Nouveaux cahiers de la recherche en éducation

\title{
Avantages et limites des approches méthodologiques utilisées pour étudier les pratiques enseignantes
}

\section{Marie Dupin de Saint-André, Isabelle Montésinos-Gelet et Marie-France Morin}

Volume 13, numéro 2, 2010

URI : https://id.erudit.org/iderudit/1017288ar

DOI : https://doi.org/10.7202/1017288ar

Aller au sommaire du numéro

Éditeur(s)

Faculté d'éducation, Université de Sherbrooke

ISSN

1911-8805 (numérique)

Découvrir la revue

Citer cet article

de Saint-André, M. D., Montésinos-Gelet, I. \& Morin, M.-F. (2010). Avantages et limites des approches méthodologiques utilisées pour étudier les pratiques enseignantes. Nouveaux cahiers de la recherche en éducation, 13(2), 159-176. https://doi.org/10.7202/1017288ar
Résumé de l'article

Dans les recherches en sciences de l'éducation, les pratiques enseignantes semblent être de plus en plus choisies comme objet d'investigation. Or, ces pratiques peuvent être examinées au moyen de différentes approches méthodologiques (Bru, 2002a). À partir des écrits sur ce sujet, nous proposons une synthèse des avantages et des limites des approches méthodologiques qui paraissent être les plus souvent utilisées pour étudier les pratiques enseignantes. Dans cette optique, nous avons regroupé ces approches en trois grandes familles en fonction de leur visée : les approches à visée prescriptive, les approches à visée heuristique et les approches à visée pratique. 


\title{
Avantages et limites des approches méthodologiques utilisées pour étudier les pratiques enseignantes
}

\author{
Marie Dupin de Saint-André, Isabelle Montésinos-Gelet
}

Université de Montréal

et Marie-France Morin

Université de Sherbrooke

\section{Résumé}

Dans les recherches en sciences de l'éducation, les pratiques enseignantes semblent être de plus en plus choisies comme objet d'investigation. Or, ces pratiques peuvent être examinées au moyen de différentes approches méthodologiques (Bru, 2002a). À partir des écrits sur ce sujet, nous proposons une synthèse des avantages et des limites des approches méthodologiques qui paraissent être les plus souvent utilisées pour étudier les pratiques enseignantes. Dans cette optique, nous avons regroupé ces approches en trois grandes familles en fonction de leur visée: les approches à visée prescriptive, les approches à visée heuristique et les approches à visée pratique.

\section{Abstract}

Research in Education Science is increasingly selecting teaching practices as subject of inquiry. However, these practices can be studied with different methodological approaches (Bru, 2002a). Based on literature written on the subject, we propose a summary of the benefits and limits of the methodological approaches seemingly most often used to study teaching practices. We have therefore grouped these approaches in three large families according to their purpose: prescriptive approaches, heuristic approaches and practical approaches.

\section{Resumen}

En las investigaciones en Ciencias de la Educación, las prácticas docentes parecen ser elegidas cada vez más como objeto de investigación. Ahora bien, se puede analizar estas prácticas por medio de varios enfoques metodológicos (Bru, 2002a). Partiendo de escritos sobre el tema, proponemos una síntesis de las ventajas y de los límites de los enfoques metodológicos que parecen ser más utilizados al momento de estudiar las prácticas docentes. En esta óptica, hemos agrupado estos enfoques en tres grandes familias según su objetivo: los enfoques con objetivo prescriptivo, los enfoques con objetivo heurístico y los enfoques con objetivo práctico. 


\section{Introduction}

Les pratiques enseignantes constituent un objet d'étude privilégié dans les recherches en sciences de l'éducation. Diverses approches méthodologiques permettent de les appréhender (Bru, 2002a), lesquelles se différencient notamment par leur visée, par leurs méthodes de collecte et de traitement des données, ainsi que par leurs retombées. Le choix d'une approche méthodologique dépend, d'une part, des objectifs et de l'orientation théorique de la recherche et, d'autre part, des avantages et des limites de chaque approche. Nous tentons ici de faire une synthèse des avantages et des limites des approches méthodologiques les plus communément utilisées dans les recherches sur les pratiques enseignantes. Nous regroupons ces approches en trois grandes familles, en fonction de leur visée: les approches à visée prescriptive, celles à visée heuristique et celles à visée pratique.

\section{Quelques clarifications conceptuelles}

Deux clarifications conceptuelles semblent préalables à notre analyse : qu'est-ce que la pratique enseignante? Qu'est-ce qu'une approche méthodologique?

\subsection{La pratique enseignante: une ou des définitions?}

Le terme "pratique enseignante", qui au premier abord semble simple, ne revêt pas la même signification pour tous les auteurs. Il est même parfois considéré comme un concept flou (Bressoux, 2001, p. 44). Nous préférons dire qu'il est polysémique (Tupin, 2003) et que son sens dépend des orientations de la recherche (Altet, 2003). En effet, prise dans son sens le plus restreint, la pratique enseignante est parfois uniquement associée à une partie de la pratique d'enseignement: la mise en œuvre d'une méthode d'enseignement (Oliveira, 2005). Tandis que dans son acception la plus large, la pratique enseignante peut être considérée en tant que système composé de sous-systèmes : «les pratiques d'enseignement [...], les pratiques formalisées entre enseignants [...], les pratiques pendant les temps informels », etc. (Marcel dans Bru et Talbot, 2001, p. 11). Compte tenu de cette variabilité du terme, nous n'en donnons pas une définition à priori, mais nous présentons, pour chaque approche méthodologique, la vision de la pratique enseignante qui lui est généralement associée.

Dans les recherches, il est fréquent, que seule la pratique d'enseignement soit prise en compte. Par conséquent, il nous semble nécessaire de la définir. La pratique d'enseignement "se déroule durant le temps scolaire, principalement en classe, en présence d'élèves » (Deaudelin, Lefebvre, Brodeur, Mercier, Dussault et Richer, 2005, p. 83). Elle comprend trois phases: préactive (la planification de l'enseignement), interactive (en classe avec les élèves) et postactive (les activités d'évaluation et de retour sur l'action) (Bressoux, 2002). Au cours de notre présentation des approches, nous ferons référence à ces différentes phases.

\subsection{La notion d'approche méthodologique}

Dans les écrits scientifiques consultés, aucune définition précise du terme "approche méthodologique» n’était proposée. Cependant, nous avons constaté qu'il se distingue de celui 
de «méthode». En effet, la méthode renvoie aux moyens utilisés pour collecter et analyser les données, tandis que la notion de méthodologie, plus large, est étroitement liée aux choix théoriques et épistémologiques (Savoie-Zajc et Karsenti, 2004). Ainsi, elle ne peut être réduite aux méthodes de collecte et d'analyse de données. Plus précisément, ces méthodes «ne constituent pas en ellesmêmes la méthodologie, mais bien l'opérationnalisation des choix méthodologiques» (Ibid., p. 113). En ce sens, dans cet article, le terme "approche méthodologique» renvoie à l'ensemble de la démarche utilisée pour appréhender les pratiques enseignantes: du choix de l'échantillon jusqu'aux retombées de la recherche. Puisque cette démarche est influencée par les choix théoriques et épistémologiques, nous serons parfois amenée à considérer ces éléments.

Afin de catégoriser les approches méthodologiques utilisées pourétudier les pratiques enseignantes, nous nous sommes inspirée de trois typologies, les ordonnant en fonction de leur visée (Bru, 2002a; Marcel, Olry, Rothier-Bautzer et Sonntag, 2002; Lebrun, Lenoir, Oliveira et Chalghoumi, 2005). Ainsi, dans un premier temps, nous présentons les approches méthodologiques à visée prescriptive; puis, nous nous intéressons à celles à visée heuristique et, enfin, nous traitons de celles à visée pratique.

\section{Les approches méthodologiques à visée prescriptive}

Les approches méthodologiques à visée prescriptive sont utilisées dans le but d'identifier les pratiques enseignantes les plus efficaces. Elles reposent sur un paradigme positiviste selon lequel la réalité, qui «existe indépendamment» de celui qui l'observe, peut être morcelée en variables qui sont observables et mesurables (Savoie-Zajc et Karsenti, 2004, p. 115). Au sein de cette catégorie, il est possible de dégager deux types d'approches méthodologiques: les approches de type «processus-produit» et celles qui visent à évaluer les pratiques enseignantes.

\subsection{Les approches de type "processus-produit"}

Dans ce type d'approche, la pratique enseignante est réduite aux comportements (effectifs ou déclarés) de l'enseignant en classe. Les caractéristiques du maître par rapport à sa pratique sont aussi prises en compte (ex., le nombre d'années d'expérience). Ses caractéristiques et ses comportements en classe (les processus) représentent les variables qui vont servir à expliquer les variations de résultats des élèves (les produits) (Altet, 2003). En raison de la relation processusproduit qui peut être rapprochée de l'association stimulus-réponse, ces approches sont souvent associées au courant théorique béhavioriste (Ibid.). Il ne s'agit pas de recherches avec un dispositif expérimental dans lequel une variable indépendante est manipulée, mais plutôt de recherches descriptives à tendance explicative (Gagné, Lazure, Sprenger-Charolles, Ropé, 1989). Le but est d'établir une relation de cause à effet (causalité linéaire) entre les comportements des enseignants et les résultats des élèves afin d'identifier les comportements efficaces.

Dans ce genre d'approche méthodologique, les échantillons doivent suffisamment grands pour être le plus représentatifs possible de la population (Fortin, 2006; Suchaut, 2003).

Plusieurs types de méthode de collecte de données sont associés à cette approche afin d'étudier les comportements des maîtres qui sont, soit identifiés à l'aide d'un questionnaire, soit pris en note à partir d'une observation en classe (Bressoux, 2002). 
Le questionnaire comporte généralement une grande majorité de questions fermées. Son utilisation présente quelques avantages. Tout d'abord, il permet de faire une enquête sur un territoire étendu (Bressoux, 2001; Fortin, 2006). Puis, sa passation et son traitement sont rapides et peu coûteux (Charron, 2004). Pour certains, le fait que l'enseignant puisse y répondre de façon autonome et anonyme tend à l'amener à produire des réponses sincères (Allaire, 1988), alors que pour d'autres, le phénomène de «désirabilité sociale» reste présent lors de la passation d'un questionnaire écrit (Bressoux, Bru, Altet, Leconte-Lambert, 1999, p.99).

Toutefois, le questionnaire comporte aussi des limites. Premièrement, il ne permet d'accéder qu'au discours de l'enseignant surses comportements et non à ses comportements effectifs (Bressoux, 2001). Or, il existe des différences entre la pratique déclarée et la pratique observée: «d'une part, l'enseignant n'est pas toujours conscient de ce qui se passe effectivement dans sa classe; d'autre part, les discours mettent en œuvre des processus de reconstruction et de rationalisation » qui peuvent amener l'enseignant à justifier ses actions après coup (Bressoux, 2001, p. 42). Deuxièmement, une autre limite réside dans la nature même du questionnaire qui n'est pas propice à une segmentation fine des comportements des maitres et "condui[t] à une construction de variables assez grossières" ) (Bressoux et al., 1999, p. 99). Ce mode de collecte de données laisse aussi, à notre sens, peu de place à la singularité, surtout lorsque les questions sont fermées. Pour finir, puisque des travaux ont montré qu'il existe une grande variabilité inter-maîtres (Bressoux, et al., Ibid.), il est possible qu'en privilégiant des questions fermées, le chercheur omette des comportements de l'enseignant relatifs à la problématique de recherche (Fraenkel et Wallen, 2003). Afin de contrôler ce biais, il est conseillé, dans le cas des questions à choix multiples, d'introduire une catégorie "autre (à préciser)» (Fraenkel et Wallen, 2003). De plus, les questions doivent être très claires, car, en cas d'incompréhension, les répondants ne pourront pas avoir d'explication (Blais et Durand, 2003).

L'observation, quant à elle, est réalisée à l'aide d'une grille (Bressoux, 2002) qui constitue une liste des comportements plausibles de l'enseignant, lesquels se rapportent au problème de recherche (Mialaret, 2004). L'observateur doit noter les comportements présents et leur fréquence (Bressoux, ibid.). Parmi les recherches consultées, même si l'importance d'avoir recours à de telles observations était soulevée (Bressoux, 2001), seuls les questionnaires avaient été utilisés. Pour cette raison, nous ne traitons pas des avantages et des limites de l'observation en tant qu'outil de collecte de données.

Dans ces approches, les performances des élèves, la plupart du temps en langue maternelle et en mathématiques, sont évaluées à l'aide de tests standardisés (Bressoux, 2002), ce qui offre l'avantage de situer les élèves au regard des résultats normaux attendus pour cet âge.

Les données ainsi obtenues sur les comportements des maitres et sur les performances des élèves font l'objet d'une analyse statistique inférentielle. Au début de ces approches "processus-produit», il s'agissait d'établir des corrélations entre les comportements des enseignants et les résultats des élèves. Ensuite, l'évolution des modèles statistiques, avec l'avènement des analyses statistiques multi-niveaux, a permis de considérer simultanément un plus grand nombre de variables et de déceler des effets-maîtres (Bru, 2002a). Il s'agit, dans ce cas, d'utiliser des modèles de régression multiple qui présentent l'avantage de pouvoir prendre en compte les caractéristiques des élèves (ex., le milieu socio-économique) de façon à «raisonner toutes choses égales par ailleurs» (Bressoux, 2001, p. 38). 
L'avantage de ce type de traitement de données est qu'il permet d'identifier des comportements des enseignants qui peuvent être des facteurs explicatifs des résultats des élèves. Une des limites de ce traitement des données est que de nombreuses conditions d'application doivent être respectées afin de pouvoir faire une analyse statistique de régression linéaire multiple. De plus, attendu que le nombre de variables explicatives pouvant être incluses dans le modèle dépend du nombre de sujets de l'échantillon, il est nécessaire d'avoir un échantillon de grande taille.

\section{Les avantages et les limites des approches de type «processus-produit»}

L'avantage majeur des approches de type "processus-produit» est qu'elles ont permis de souligner le rôle important de la pratique de l'enseignant dans la progression des élèves et d'identifier des variables considérées comme efficaces (Altet, 2003).

Par contre, les analyses dans ces approches font abstraction du contexte de réalisation des comportements du maître et considèrent que la pratique enseignante est réductible à certains comportements. En effet, ces approches restreignent les pratiques enseignantes à un nombre limité de variables et ne donnent qu' " une explication en termes de causalité linéaire » (Clanet, dans Bru et Talbot, 2001, p. 13), c'est-à-dire qu'une cause (l'enseignement) provoque un effet (l'apprentissage) (Clanet, 2002). En ce sens, il est nécessaire de préciser que tous les apprentissages des élèves ne sont pas attribuables à l'enseignement reçu en classe (ibid.). Une autre limite de ces approches est que le rôle joué par l'élève dans l'enseignement-apprentissage n'est pas pris en compte (Altet, 2003). Pour finir, un autre désavantage des résultats obtenus par ces approches réside dans leur utilisation et leur généralisation. En effet, «on peut souhaiter que l'application de la modalité réputée efficace soit généralisée, mais il reste à s'interroger sur les conditions de cette application (validité écologique)»(Bru, 2002a, p. 65). Ainsi, la validité statistique des résultats ne leur confère pas une validité pratique (Bru, 2002b).

\subsection{Les approches visant à évaluer les pratiques enseignantes}

Dans ce type d'approche, la pratique enseignante est réduite à la mise en œuvre d'une méthode d'enseignement (Altet, 2003). Étudier la pratique enseignante revient alors à évaluer les effets de la méthode d'enseignement utilisée par l'enseignant sur les apprentissages des élèves. Pour ce faire, des dispositifs de recherche expérimentaux, ou quasi-expérimentaux, sont employés. Ce dispositif de recherche permet de distinguer cette approche de celle de type «processus-produit».

Dans une recherche expérimentale, il s'agit de manipuler une variable indépendante (méthode d'enseignement) et d'en observer les effets sur une variable dépendante (les habiletés des élèves). Pour ce type de recherche, quatre conditions doivent être respectées : choix de l'échantillon, au hasard, au sein de la population concernée; mise en place d'un groupe contrôle; répartition aléatoire des sujets dans les groupes expérimentaux (utilisateurs de la méthode d'enseignement) et contrôles (non-utilisateurs de la méthode d'enseignement); et équivalence des groupes expérimentaux et contrôles (Boudreault, 2004). Dans les recherches en éducation, il semble moins facile de respecter ces conditions d'expérimentation. Ainsi, les dispositifs de recherche sont, le plus souvent, quasiexpérimentaux, c'est-à-dire qu'ils respectent uniquement la condition de présence d'un groupe contrôle (Boudreault, 2004). Bien que cette approche vise à évaluer les pratiques enseignantes, 
celles-ci ne font habituellement pas, dans ce cas, l'objet d'une collecte de données. Il s'agit, le plus souvent, d'évaluer les performances des élèves. Ainsi, les élèves des groupes expérimentaux et contrôles participent à un prétest et à un post-test au cours desquels leurs habiletés relatives à l'objet étudié sont évaluées.

Lors de l'évaluation des performances des élèves, les tests d'habiletés standardisés sont souvent privilégiés. Par contre, de tels tests ne sont pas disponibles pour toutes les habiletés qui peuvent faire l'objet d'une évaluation. Dans ce cas, le chercheur doit créer ou adapter des épreuves pour évaluer l'habileté concernée. L'avantage est alors qu'il peut choisir avec discernement précisément ce qui sera évalué. Le désavantage en sera que ce choix peut impliquer l'omission de données qui pourraient être importantes. Toutefois, il ne possède pas alors de données quant aux résultats attendus pour un âge donné et n'a pas autant de garantie qu'un test standardisé en ce qui a trait à la

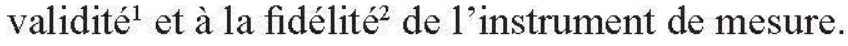

Le traitement des données consiste en une comparaison statistique des résultats obtenus au post-test par les groupes expérimentaux et les groupes contrôles afin d'identifier l'effet produit par la variable indépendante sur la variable dépendante. Parfois, il s'agit aussi de comparer la progression des élèves des deux groupes entre le prétest et le post-test.

L'avantage d'un tel traitement quantitatif des données est qu'il permet de comparer des groupes et de vérifier si les différences entre eux sont statistiquement significatives. Par contre, le fait que les groupes ne soient pas constitués au hasard est un biais qui vient entacher la possibilité de les comparer (Van der Maren, 1996). De plus, comme nous 1'avons déjà précisé, l'emploi de tests statistiques demande de respecter des conditions d'application. Un autre avantage du traitement statistique est qu'un résultat, considéré statistiquement significatif, peut être généralisé à l'ensemble de la population de la recherche à condition qu'une certaine rigueur méthodologique soit respectée.

\section{Les avantages et les limites des approches visant à évaluer les pratiques enseignantes}

Ces approches présentent l'avantage de pouvoir «évaluer» l'impact de méthodes d'enseignement sur les résultats des élèves dans des classes. Par contre, ce type d'approche, parfois intitulée « entrée par les méthodes » (Altet, 2003, p. 33), présente plusieurs limites. Premièrement, il réduit la pratique enseignante à la seule application d'une méthode d'enseignement (Altet, 2003). L'enseignant tend alors à être considéré comme un technicien qui applique à la lettre une méthode (Lebrun et al., 2005). Deuxièmement, le fait que ne soit considéré que l'absence ou la présence de la méthode d'enseignement rend difficile 1'application des résultats dans la pratique. En effet, la façon dont les enseignants emploient la méthode est rarement prise en compte. Pourtant, il existe une grande variabilité de comportements reliés à l'enseignement chez les maîtres (Bressoux et al., 1999). Pour cette raison, Bru (1998) remet en cause la fidélité de ce type de recherche puisque chaque enseignant applique, à sa manière, la méthode dans sa classe. De façon à dépasser cette limite, il est possible d'utiliser un questionnaire pour recueillir les pratiques déclarées des enseignants quant à l'utilisation de la méthode, ou de les observer en train de la mettre en cuvre.

1 «La validité est associée au fait de bien mesurer ce qu'on veut mesurer.» (Boudreault, 2004, p. 171).

2 «La fidélité d'un instrument dénote sa capacité de toujours mesurer la même chose, c'est-à-dire que dans des circonstances semblables, quel que soit l'évaluateur, des résultats similaires devraient être obtenus.» (Ibid.) 


\subsection{Synthèse des approches méthodologiques à visée prescriptive}

Ces deux types d'approches à visée prescriptive privilégient l'utilisation de méthodes de recherche quantitatives qui offrent, à partir d'une vision segmentée de la pratique enseignante en variables, des résultats sur le plan de l'efficacité. L'accent est majoritairement mis sur l'étude des pratiques d'enseignement, réduites aux comportements des maîtres et/ou à la mise en œuvre d'une méthode, et sur leurs impacts sur les performances des élèves. L'élève n'est pas considéré comme un élément actif du processus d'enseignement-apprentissage. De plus, ces approches fournissent des résultats au rayonnement limité quant à leur utilité pour les enseignants.

Les limites de ces approches, quant au peu d'informations qu'elles fournissent sur les pratiques enseignantes, ont amené les chercheurs à mettre en place de nouvelles approches méthodologiques davantage axées sur l'étude approfondie des pratiques enseignantes.

\section{Les approches méthodologiques à visée heuristique}

Plusieurs chercheurs soulèvent la nécessité de développer des connaissances sur les pratiques enseignantes avant d'être en mesure de les évaluer (Bressoux et al., 1999; Bru, 2002a; Altet, 2003). Les approches utilisées à cette fin sont de nature descriptive comme l'enquête (par questionnaire et par entretien) ou l'étude de cas. Ainsi, il est question de décrire les pratiques enseignantes dans le but de pouvoir, dans un premier temps, les comprendre et, dans un temps ultérieur, les expliquer.

\subsection{Les enquêtes sur les pratiques enseignantes}

L'enquête permet d'appréhender de multiples dimensions de la pratique enseignante à travers les pratiques déclarées par l'enseignant (ex. les croyances des enseignants face à un objet d'enseignement).

L'enquête par questionnaire porte généralement sur un échantillon de grande taille choisi de façon aléatoire au sein de la population de recherche afin qu'il soit représentatif de cette population (Gagné et al., 1989; Fortin, 2006). Le questionnaire peut comporter des questions fermées et/ou ouvertes. En raison du faible coût associé à sa passation et à son traitement, cet outil est souvent privilégié dans les recherches sur les pratiques enseignantes (Charron, 2004). Toutefois, un des désavantages du questionnaire est que, parfois, le nombre de répondants reste peu élevé (Blais et Durand, 2003). Afin de contrôler cette limite, l'envoi d'avis de rappels aux non-répondants peut permettre d'augmenter le taux de réponse ( $I$ bid.). Pour finir, cette méthode comporte le risque que certaines questions soient laissées de côté par les répondants] (Fortin, 2006).

Les données issues des questionnaires qui comportent des questions fermées vont être traitées à l'aide de statistiques descriptives (Gauthier, 2003). Il s'agit entre autres de donner des indications de fréquence, de tendance centrale et de dispersion (Charbonneau, 1988).

Il est fréquent que l'enquête par questionnaire soit complétée avec une enquête par entretien. Dans cette dernière, il s'agit de choisir parmi l'échantillon initial un nombre restreint de sujets, afin d'étudier de manière plus approfondie leurs pratiques. 
Il existe deux modalités de passation de l'entretien, par téléphone ou en face à face (Fortin, 2006), et il se décline sous plusieurs formes, dirigé avec des questions fermées, préparées à l'avance et posées dans un ordre prédéterminé ou semi-dirigé ${ }^{3}$ avec des questions ouvertes, préparées à partir des thèmes à aborder au cours de la rencontre, et posées dans l'ordre considéré comme le plus à propos pendant l'entrevue (Ibid.). Ces questions ouvertes sont pertinentes lorsque le but de la recherche est d'obtenir des réponses personnelles (Blais et Durand, 2003). Par contre, ces questions, de par leur singularité, sont plus difficiles à traiter que les questions fermées (Fraenkel et Wallen, 2003). Les données ainsi obtenues seront retranscrites et feront l'objet d'une analyse de contenu (Fortin, 2006).

Un des points forts de l'entretien en face à face est qu'il facilite l'établissement d'une relation de confiance (Charron, 2004). En ce sens, il est nécessaire de fournir à l'enseignant préalablement à la rencontre, un document qui lui donnera des explications quant au déroulement de l'entrevue, à sa durée et aux informations qui lui seront demandées (Van der Maren, 1999). Un deuxième avantage de l'entretien concerne la compréhension des questions, puisque l'enseignant peut obtenir des clarifications en cas d'incompréhension (Charron, 2004). Une limite de l'entretien est le phénomène de «désirabilité sociale» (Allaire, 1988) qui risque d'amener l'enseignant à donner la réponse qu'il pense adéquate en fonction des attentes du chercheur, afin que celui-ci génère une image positive de lui.

L'entretien par téléphone présente l'avantage d'être relativement peu coûteux, rapide et permet de questionner des personnes qui résident dans des régions éloignées (Blais et Durand, 2003). Cependant, il peut être dans ce cas plus difficile d'établir une relation de confiance avec l'enseignant (Fortin, 2006).

Pour que des approches méthodologiques d'enquête soient valides, il faut que les individus de l'échantillon soient disponibles pour répondre à l'enquête, qu'ils disposent de l'information recherchée, qu'ils la transmettent avec un minimum de distorsion et que cette information soit consignée avec rigueur (Blais et Durand, 2003). Par contre, une des limites de ces approches méthodologiques est qu'elles permettent seulement d'accéder aux pratiques déclarées de l'enseignant (Charron, 2004). Afin de contrer ce biais, il est conseillé de demander à l'enseignant des traces de la mise en œuvre de ses pratiques (ex., des travaux d'élèves [Ibid.]).

En somme, les enquêtes permettent de faire une macro-analyse des pratiques enseignantes en privilégiant «un point de vue global, valable pour un grand nombre» (Terrisse, 2002, p. 244). Par contre, elles ne donnent pas la possibilité de décrire finement les pratiques enseignantes. Pour ce faire, il est important d'avoir recours à des études de cas.

\subsection{Les études de cas sur les pratiques enseignantes}

L'étude de cas ne semble pas revêtir le même sens pour tous les auteurs (Ndouna Nsondé, 2005). Dans cet article, l'étude de cas est définie comme une approche méthodologique (Roy, 2003) qui «consiste en l'examen détaillé [...] d'un phénomène lié à une entité sociale qui peut

3 Nous mentionnerons un autre type d'entretien (non dirigé) lorsque nous traiterons de l'étude de cas. 
être un individu, un groupe, une famille, une communauté ou une organisation» (Fortin, 2006, p. 192). Il est possible d'étudier la pratique enseignante d'un ou de plusieurs individus, (nombre restreint) dans «une étude de cas simple», ou bien "une étude de cas multiples» (Van der Maren, 1996, p. 238). La deuxième forme d'étude de cas diffère de la première, car les cas sont étudiés dans le but d'être comparés. Selon leur affiliation théorique, les auteurs opteront pour le choix d'un ou plusieurs cas, et mettront en place un dispositif de collecte de données approprié. Il est important de noter que "l'étude de cas est par définition éclectique au sens où toutes les approches théoriques et toutes les méthodes de constitution de données qui peuvent fournir des éléments aidant à comprendre la complexité du phénomène devront être envisagées " (Van der Maren, 1996, p. 238). Compte tenu de cette diversité, nous nous limitons à la description des méthodes de collecte et d'analyse de données les plus fréquemment utilisées.

Dans 1'étude de cas, il ne s'agit pas de choisir un échantillon représentatif de la population, mais plutôt de sélectionner peu de participants et d'en faire une analyse approfondie. Ce choix des participants, qui est non probabiliste, peut se faire de différentes façons (pour une description approfondie de ces techniques d'échantillonnage, voir Fortin [2006]). Le nombre de participants est souvent fonction de la profondeur de l'analyse du cas prévu. Dans les études de cas, les méthodes de collecte de données le plus souvent utilisées sont l'observation et l'entretien (semi-dirigé ou non dirigé) (Ibid., p. 192).

Dans les écrits consultés, l'observation des pratiques enseignantes portait principalement sur la phase au cours de laquelle l'enseignant est en présence des élèves. Toutefois, les éléments observés au sein de la pratique varient en fonction des objectifs de recherche et du cadre conceptuel.

L'observation peut se décliner sous quatre formes: 1) la participation observante qui consiste en une observation menée par un enseignant au sein de sa propre pratique d'enseignement; 2) l'observation participante où une personne s'insère et participe à la vie de la classe tout en observant et en prenant en note ce qui s'y passe; 3) l'observation systématique, où un observateur note ce qui se déroule en classe; elle est dite directe lorsqu'elle est faite sans grille d'observation; 4) l'observation électronique qui consiste à enregistrer, ou à filmer, la pratique d'enseignement (Van der Maren, 1999, p. 137). En éducation, les deux premiers types d'observation sont nettement moins fréquents. Ainsi, nous nous limitons à la présentation des observations systématique, (avec grille ou directe) et électronique.

Lors de l'observation systématique, il s'agit d'observer, à l'aide d'une grille pré-établie, quelques variables dans les pratiques d'enseignement pour ensuite les quantifier (Marcel et al., 2002). Lors de l'observation directe sans grille, l'observateur note tout ce qui lui apparaît pertinent au regard de sa recherche (Savoie-Zajc, 2004). Ce type d'observation peut être combiné avec une observation électronique. L'observation électronique permet d'appréhender la pratique d'enseignement dans sa globalité. Ainsi, les observations filmées, ou enregistrées, peuvent faire l'objet d'une analyse minutieuse (Beaugrand, 1988; Marcel et al., 2002). Ce deuxième type d'observation peut être accompagné d'un entretien «d'auto-confrontation» au cours duquel 1'enseignant visionne l'enregistrement avec le chercheur et commente ses comportements (Goigoux, 2002). Cet entretien sert à extraire le sens que l'enseignant donne à sa pratique. 
L'avantage majeur de l'observation est qu'elle donne la possibilité d'accéder à ce qui se passe réellement en classe (Charron, 2004) et d'en faire une description riche. De plus, elle permet au chercheur de prendre connaissance de certains éléments de la pratique d'enseignement qui n'auraient pas pu être mis au jour autrement (Fortin, 2006). Comparativement à l'observation en direct, l'observation filmée fournit l'avantage de pouvoir être visionnée à plusieurs reprises de façon à pouvoir en affiner sa description (Beaugrand, 1988). De plus, l'observation électronique permet de prendre en compte le contexte dans lequel les comportements se manifestent (Van der Maren, 1999).

L'observation, qu'elle soit systématique ou électronique, comporte aussi des limites. En effet, il peut y avoir «un effet d'intrusion de l'observateur» (Beaugrand, 1988), c'est-à-dire que la présence de l'observateur, ou de la caméra, peut amener l'enseignant et les élèves à modifier leurs comportements. Pour cette raison, nous préférons au terme "pratique effective» celui de "pratique constatée» (Bru, 2002a) qui différencie la pratique habituelle de l'enseignant de celle observée par le chercheur. Pour finir, l'observation est une méthode de collecte de données qui est coûteuse en temps et en argent (Charron, 2004). Lors des observations systématiques, il existe également des limites liées à la grille d'observation et à son emploi. En effet, il est possible que, lors de sa construction, le chercheur ait omis des comportements (Van der Maren, 1999). De plus, si elle est employée par plusieurs personnes, chaque individu peut en avoir sa propre compréhension (Ibid.). Afin de contrôler cette limite, il peut être intéressant d'introduire des définitions des comportements et des exemples de ceux-ci dans la grille. De plus, afin d'augmenter la fidélité des observations, une formation peut être donnée aux futurs observateurs (Ibid.).

Les entretiens utilisés dans les études de cas sont généralement semi-dirigés ${ }^{4}$ ou non dirigés ${ }^{5}$. Au cours de l'entretien non dirigé, l'enseignant parle de sa pratique à partir de questions ouvertes dont l'ordre n'est pas déterminé à l'avance. Les entretiens peuvent porter sur toutes les phases de la pratique d'enseignement. De plus, des entretiens peuvent aussi être menés sur d'autres thèmes relatifs à la pratique enseignante puisque celle-ci ne se limite pas à la pratique d'enseignement (Altet, 2003).

Un des avantages de l'entretien semi-dirigé, ou non dirigé, est qu'il permet d'obtenir des données détaillées sur les pratiques enseignantes (Savoie-Zajc, 2003). Par contre, étant donné le risque d'effet de "désirabilité sociale», il est pertinent d'utiliser la triangulation des données, c'est-à-dire de jumeler l'entretien à d'autres types de collecte de données (ex., le journal de bord de l'enseignant) pour assurer la crédibilité ${ }^{6}$ des données obtenues (Ibid.).

Quant à l'entretien «d'auto-confrontation», il présente l'avantage de permettre de connaître la signification que l'enseignant attribue à sa pratique d'enseignement.

4 Nous ne reprenons pas la description des entretiens semi-dirigés présentée précédemment.

5 Nous tenons à mentionner une forme particulière d'entretien non dirigé de plus en plus utilisée dans les sciences de l'éducation: l'instruction au sosie. La mise en situation est la suivante: «suppose que je sois ton sosie et que demain je me trouve en situation de te remplacer dans ton travail. Quelles sont les instructions que tu devrais me transmettre afin que personne ne s'avise de la substitution?» (Saujat, 2002, p. 109).

6 Assurer la crédibilité dans une recherche qualitative consiste à s'assurer que «le sens attribué au phénomène est plausible et corroboré par diverses instances» (Savoie-Zajc, 2004, p. 143). 
Les données issues des observations systématiques sont traitées à l'aide d'analyses statistiques descriptives. Les données obtenues lors des observations directes et filmées ainsi que les données issues des entretiens sont soumises à une analyse de contenu. Il s'agit alors de découper le verbatim (c'est-à-dire la retranscription des observations) en unités de sens, qui sont ensuite codées ${ }^{7}$. Pour finir, ces données codées pourront faire 1'objet d'un traitement qualitatif ou quantitatif (Van der Maren, 1996).

Nous avons vu qu'un moyen de contrôler les biais dans l'étude de cas était la triangulation des données. Or, il est aussi possible de faire "une triangulation par l'analyse» (Savoie-Zajc, 2004, p. 147) dans laquelle les mêmes données sont analysées de différentes manières, afin d'en enrichir la compréhension. En ce sens, Altet (2002) privilégie «une analyse plurielle» dans laquelle des chercheurs issus de différentes disciplines, considérées comme contributives des sciences de l'éducation ${ }^{8}$, vont analyser une même observation et confronter leur point de vue. Le but de cette analyse est d'identifier et de comprendre les composantes de la situation d'enseignement observée (Altet, 2002) pour en faire une modélisation (Marcel, et al., 2002).

Dans le cas où les données sont obtenues au moyen de l'observation et de l'entretien, ces données sont comparées et confrontées. Ainsi, «cette double lecture ${ }^{9}$ " (Marcel, 2002) permet au chercheur d'avoir une compréhension plus complète de la pratique de l'enseignant.

Le principal avantage de ce type de traitement des données est qu'il permet de faire une analyse en profondeur des pratiques enseignantes. Par contre, il est très coûteux en temps et en argent. De plus, compte tenu de la subjectivité du chercheur lors de l'analyse de contenu, il est nécessaire de procéder à «un codage multiple» pour vérifier la fiabilité du codage (Miles et Huberman, 2003).

En résumé, l'étude de cas, en tant qu'approche de l'ordre de la micro-analyse, permet de procéder à une description détaillée des pratiques enseignantes, notamment au moyen de l'observation qui donne la possibilité d'accéder à ce qui se déroule dans la salle de classe. Cette approche permet de développer des connaissances sur des sujets peu connus (Roy, 2003). Une de ses limites est qu'elle s'intéresse à peu d'individus et n'est donc pas représentative de la population ( $I$ bid.). Ainsi, il n'y pas de possibilité de généralisation des résultats obtenus (Terrisse, 2002).

\subsection{Synthèse des approches méthodologiques à visée heuristique}

Les approches méthodologiques à visée heuristique visent à décrire au moyen d'une macroanalyse (les enquêtes) ou d'une micro-analyse (les études de cas) les pratiques enseignantes. Contrairement aux deux approches méthodologiques à visée prescriptive qui étudient la pratique

7 Il existe trois types de codages: fermé (utilisation d'une grille fermée construite à partir du cadre conceptuel), ouvert (sans grille) et mixte (avec une grille dans laquelle des codes peuvent être ajoutés) (Van der Maren, 1996, p. 436). Ces codages reposent sur des positions épistémologiques différentes et sont représentatifs de la controverse qui existe quant à «la place du cadre théorique dans l'analyse de données qualitatives » (Savoie-Zajc, 2004, p. 139).

8 Il s'agit pour cette auteure de l'épistémologie, de la pédagogie, de la didactique, de la psychologie et de la sociologie (Altet, 2002).

9 Cette double lecture comporte « une lecture extrinsèque privilégiant les comportements observables et une lecture intrinsèque privilégiant l'explicitation des significations de l'enseignant» (Lefeuvre, 2005, p. 82). 
enseignante d'une façon très similaire, dans les approches à visée heuristique les pratiques enseignantes sont appréhendées de différentes façons. À ce propos, il faut noter que nous avons présenté les approches à visée heuristique de façon très cloisonnée. Or, il existe de plus en plus de recherches «hybrides» qui combinent différentes approches méthodologiques (enquête et étude de cas) et diverses méthodes de collecte et de traitement des données, afin d'avoir une connaissance plus approfondie des pratiques enseignantes (pour une recherche de ce type, voir Lenoir [2006]).

En résumé, les approches méthodologiques à visée heuristique permettent au chercheur de saisir la richesse des pratiques enseignantes (Tupin, 2003) et ainsi d'en développer une meilleure connaissance sans chercher à les évaluer. Toutefois, puisque l'accent est mis sur la description des pratiques enseignantes, les résultats ne permettent pas de porter un jugement quant à l'efficacité deces pratiques (Tupin, 2003). De plus, une des limites de ces approches est que leurs retombées sont essentiellement utiles au milieu de la recherche et non à celui des praticiens. Cette limite est prise en compte dans les approches qui visent à résoudre des problèmes issus de la pratique.

\section{Les approches méthodologiques à visée pratique}

Les recherches en éducation ont longtemps été critiquées, et le sont encore, pour leur manque d'utilité pour les enseignants (Van der Maren, 1996). La mise en place d'approches méthodologiques à visée plus pratique ambitionne de combler cette lacune. Ces approches visent à transformer les pratiques enseignantes tout en les étudiant. Dans cette section, nous nous limitons à la description de deux approches : la recherche-action et la recherche collaborative ${ }^{10}$. Cette partie se démarquera, dans sa forme, des deux précédentes puisque «la recherche-action [et la recherche collaborative] ne se distingue[nt] pas des autres types de recherche par des techniques ou des méthodes spécifiques, mais plutôt par [leur] objet et le rôle du chercheur" (Dolbec et Clément, 2004, p. 195). Ainsi, nous ne décrivons pas les avantages et les limites des méthodes de collecte et de traitement des données de ces approches, mais axons plutôt notre discussion autour de la description, de la critique, de la démarche et des spécificités de la recherche-action et de la recherche collaborative.

\subsection{La recherche-action}

La recherche-action peut prendre différentes formes (Savoie-Zajc, 2001). De plus, selon sa forme, elle peut se baser sur des paradigmes distincts : "positiviste, interprétatif et critique " (Ibid., p. 25). Compte tenu de cette diversité au sein d'un même type de recherche, nous nous limitons aux caractéristiques communes aux différentes recherches-actions, tout en les appliquant à la recherche sur les pratiques enseignantes. Il appert que, généralement, une recherche-action comporte les trois éléments suivants: 1) le but principal de cette approche est de transformer, dans l'optique de les améliorer, les pratiques enseignantes ; 2) les enseignants, en tant qu'acteurs du milieu, sont engagés dans la recherche; 3) «le projet se déroule selon une spirale de cycles de planification, d'action, d'observation et de réflexion" (Ibid., p. 1711).

10 La recherche collaborative s'apparente à la recherche-action. Toutefois, il nous semble pertinent de nous intéresser à cette approche pour laquelle une démarche de recherche particulière a été modélisée par Desgagné et ses collaborateurs (2001).

11 Cette auteure s'appuie sur les écrits de King et Lonnquist (1994). Nous n'avons pas pu accéder à ce texte qui, d'après Savoie-Zajc, est inédit. 
Comme l'indique son nom, la recherche-action vise à mener simultanément des actions pour résoudre des problèmes issus des pratiques enseignantes tout en étudiant par la recherche les processus de transformation des pratiques (Dolbec et Clément, 2004). La recherche-action peut porter sur les pratiques d'enseignement, ainsi que sur d'autres aspects de la pratique enseignante.

\subsection{La recherche collaborative}

La recherche collaborative semble être une approche méthodologique de plus en plus valorisée dans le domaine des sciences de l'éducation. Cette approche demande une collaboration entre enseignants et chercheurs autour d'un élément de la pratique qui sera «un objet d'investigation conjoint au carrefour des préoccupations de l'ensemble des participants» (Desgagné, 2001, p. 52). Afin de décrire la démarche à adopter dans une telle approche, Desgagné et ses collaborateurs (2001) ont créé un modèle de l'approche collaborative décrit ci-dessous.

Dans une recherche collaborative, les enseignants et les chercheurs partent d'un thème principal à partir duquel les chercheurs et les enseignants identifient «un objet de formation (pour les enseignants) et un objet de recherche (pour le chercheur)» (Desgagné et al., 2001, p. 41). Ensuite, il s'agit de mener "une activité réflexive» au cours de laquelle les chercheurs et les enseignants étudient un élément de la pratique pour lesquels ils ont un intérêt commun (Ibid., p. 37). Il s'agit en quelque sorte d'une réflexion et d'un partage de leurs expertises respectives sur le thème à l'étude. C'est à travers cette analyse réflexive que le chercheur collectera ses données tandis que les enseignants auront des éléments de formation contribuant à leur développement professionnel (Ibid., 2001). Pour finir, les retombées de la recherche doivent se faire sentir tant dans le milieu des enseignants que dans celui de la recherche.

Étant donné que les différences entre les approches de type recherche-action et celles de type recherche collaborative se situent surtout dans la démarche adoptée, nous présentons simultanément les avantages et les limites de ces deux types d'approches.

D'une part, ces approches fournissent l'avantage de pouvoir étudier les pratiques enseignantes tout en participant à leur amélioration. De plus, puisqu'il s'agit, dans le cas de la recherche-action, de partir d'un problème issu de la pratique, il existe à la fin de la recherche des retombées directes pour les enseignants. D'autre part, ces approches méthodologiques participatives sont intéressantes, car elles permettent de prendre en compte les compétences développées par les enseignants au sein de leur pratique (Desgagné et Bednarz, 2005). Pour cette raison, à l'instar de ces auteurs, nous pouvons dire que ces recherches s'inscrivent dans une démarche de professionnalisation de l'enseignement. Un autre avantage de ces approches est qu'elles permettent au chercheur d'étudier tout autant la pratique d'enseignement que les autres pratiques de l'enseignant au sein de l'école.

Toutefois, ces approches méthodologiques présentent aussi des limites. Premièrement, lorsqu'il est question d'approches à visée pratique, un des désavantages fréquemment relevé est «le risque de confusion des rôles » entre le chercheur et les praticiens (Bru, 2002a, p. 65). Afin de pallier cette limite, il est nécessaire de cibler dès le début de la recherche, les rôles dévolus à chacun par rapport aux tâches à effectuer (ex., la création du dispositif de recherche et d'activités d'enseignement) (Dolbec et Clément, 2004). Deuxièmement, une autre critique consiste à considérer que la 
partie recherche dans ces approches n'est pas aussi développée et solide que dans d'autres types d'approches (Savoie-Zajc, 2001). Afin de s'assurer de la rigueur d'une recherche-action et d'ainsi éviter cet écueil, plusieurs critères doivent être pris en compte : «le respect des valeurs et principes démocratiques ${ }^{12}$, la faisabilité ${ }^{13}$, la cohérence systémique ${ }^{14}$, la fiabilité et l'appropriation ${ }^{15}$ " (SavoieZajc, 2001, p. 37-40). Il est à noter que ces critères nous semblent aussi très pertinents dans le cadre d'une recherche collaborative.

Pour finir, en ce qui a trait aux retombées de la recherche-action, il est possible que celles-ci ne soient que temporaires et que les enseignants, une fois que les chercheurs ne sont plus sur le terrain, remettent en place leurs anciennes pratiques (Van der Maren, 1996). Toutefois, il nous semble que la démarche adoptée dans les recherches collaboratives tend à favoriser la constance des retombées tant pour l'enseignant que pour sa communauté.

En somme, les approches méthodologiques à visée pratique donnent aux enseignants et aux chercheurs l'opportunité de travailler de concert sur un problème généralement issu de la pratique des enseignants. Elles trouvent leur originalité dans le fait de simultanément promouvoir et étudier l'action. Elles s'inscrivent dans la dynamique actuelle de professionnalisation de l'enseignement (Desgagné et Bednarz, 2005) qui tend à faire reconnaître "les savoirs d'expérience ${ }^{16}$ » de l'enseignant (Tardif et Lessard, 1999, p. 370). De plus, elles permettent d'identifier des solutions pour des problèmes issus de la pratique qui pourront potentiellement être transférées à des situations similaires (Savoie-Zajc, 2001). Toutefois, ces approches comportent également des biais, tels que la confusion entre les rôles des chercheurs et des enseignants, et le fait que les changements instaurés dans les pratiques risquent d'être temporaires.

\section{Conclusion}

L'étude des approches méthodologiques utilisées pour étudier les pratiques enseignantes nous a permis de remarquer que, bien que de nombreux chercheurs s'entendent sur le fait que la pratique enseignante ne se limite pas à la pratique d'enseignement (Altet 2003 et Lefeuvre, 2005), c'est bien souvent uniquement cette dernière qui fait l'objet d'une étude.

En guise de conclusion, nous retenons que chaque approche méthodologique amène le chercheur à adopter un point de vue particulier pour étudier les pratiques enseignantes et que «c'est bien la confrontation de ce que ces regards permettent de connaître qui présente un intérêt» (Bru, 2002a, p. 71). Ainsi, il appert que la richesse du champ de la recherche sur les pratiques enseignantes vient de cette coexistence d'approches méthodologiques distinctes et complémentaires.

12 Il s'agit de s'assurer que les valeurs, les idées, ainsi que les droits des enseignants (et des élèves) ont été respectés dans la recherche (Dolbec et Clément, 2004).

13 La faisabilité consiste à vérifier que la solution proposée correspond réellement aux besoins des participants et tient compte des contraintes du milieu (Savoie-Zajc, 2001).

14 Ce critère concerne plus la description du volet «recherche» dans la recherche-action (Ibid, 2001).

15 La fiabilité et l'appropriation font référence à la cohérence entre la problématique, la démarche choisie et les résultats obtenus (Dolbec et Clément, 2004).

16 Les savoirs d'expérience réfèrent à « un ensemble de connaissances actualisées, acquises et requises dans le cadre de la pratique du métier.» (Tardif et Lessard, 1999, p. 370) 


\section{Références}

Allaire, D. (1988). Questionnaires : mesure verbale du comportement. In M. Robert (dir.), Fondements et étapes de la recherche scientifique en psychologie ( $3^{\mathrm{e}}$ édition) (p. 229-275). Saint-Hyacinthe : Edisem. ( $1^{\mathrm{re}}$ éd. 1982)

Altet, M. (2002). L'analyse plurielle du processus enseignement-apprentissage. In J.-F. Marcel (dir.), Les sciences de l'éducation: des recherches, une discipline (p. 43-52). Paris : L'Harmattan.

Altet, M. (2003). Caractériser, expliquer et comprendre les pratiques enseignantes pour aussi contribuer à leur évaluation. Les Dossiers des Sciences de l'Éducation, 10, 31-43.

Beaugrand, J. (1988). Observation directe du comportement. In M. Robert (dir.), Fondements et étapes de la recherche scientifique en psychologie ( $3^{\mathrm{e}}$ édition) (p. 277-310). Saint-Hyacinthe : Edisem. ( $1^{\text {re }}$ éd. 1982)

Blais, A. et Durand, C. (2003). Le sondage. In B. Gauthier (dir.), Recherche sociale : de la problématique à la collecte des données (4édition) (p. 387-429). Québec: Presses de l’Université du Québec. (1 ${ }^{\text {re }}$ éd. 1984)

Boudreault, P. (2004). La recherche quantitative. In T. Karsenti et L. Savoie-Zajc (dir.), La recherche en éducation: étapes et approches (p. 151-180). Sherbrooke: Éditions du CRP.

Bressoux, P. (2001). Réflexions sur l'effet-maître et l'étude des pratiques enseignantes. Les Dossiers des Sciences de l’Éducation, 5, 35-52.

Bressoux, P. (dir.) (2002). Les stratégies de l'enseignant en situation d'interaction. Rapport de recherche pour Cognitique, Programme École et Sciences Cognitives, Ministère de la Recherche, Paris.

Bressoux, P., Bru, M., Altet, M. et Leconte-Lambert, C. (1999). Diversité des pratiques d'enseignement à l'école élémentaire. Revue française de pédagogie, 126, 97-110.

Bru, M. (1998). Qu'y a-t-il à prouver quand il s'agit d'éducation? In C. Hadji et J. Baillé (dir.), Recherche etéducation: vers une nouvelle alliance (p. 45-65). Bruxelles: De Boeck.

Bru, M. (2002a). Pratiques enseignantes: des recherches à conforter et à développer. Revue française de pédagogie, $138,63-73$.

Bru, M. (2002b). Savoirs de la recherche et savoirs des praticiens de l'enseignement. In J. Donnay et M. Bru (dir), Recherches, pratiques et savoirs en éducation (p. 133-154). Bruxelles : De Boeck.

Bru, M. et Talbot, L. (2001). Les pratiques enseignantes: une visée, des regards. Les Dossiers des Sciences de l'Éducation, 5, 9-33.

Charbonneau, C. (1988). Analyse et généralisation des résultats. In M. Robert (dir.), Fondements et étapes de la recherche scientifique en psychologie ( $3^{\mathrm{e}}$ édition) (p. 311-340). Saint-Hyacinthe : Edisem. (1 ${ }^{\mathrm{re}}$ éd. 1982)

Charron, A. (2004). La description de pratiques d'orthographes approchées d'enseignantes du préscolaire en contexte québécois : une réflexion méthodologique. In Actes du 9? colloque de l'AIRDF (p. 1-14). Québec.

Clanet, J. (2002). Gestion et organisation de l'interaction maître-élèves. In P. Bressoux (dir.), Les stratégies de l'enseignant en situation d'interaction (p. 77-108). Rapport de recherche pour Cognitique, Programme Ecole et Sciences Cognitives, Ministère de la Recherche, Paris.

Deaudelin, C., Lefebvre, S., Brodeur, M., Mercier, J., Dussault, M. et Richer, J. (2005). Évolution des pratiques et des conceptions de l'enseignement, de l'apprentissage et des TIC. Revue des sciences de l'éducation, XXI (1), $79-110$

Desgagné, S. (2001). La recherche collaborative: nouvelle dynamique de recherche en éducation. In M. Anadón (dir), Nouvelles dynamiques de recherche en éducation (p. 51-76). Québec : Les Presses de l'Université Laval.

Desgagné, S., Bednarz, N., Couture, C., Poirier, L. et Lebuis, P. (2001). L'approche collaborative de recherche en éducation. Revue des sciences de l'éducation, XXVII (1), 33-64.

Desgagné, S. et Bednarz, N. (2005). Médiation entre recherche et pratique en éducation. Revue des sciences de l'éducation, XXXI (2), 245-258. 
Dolbec, A. et Clément, J. (2004). La recherche-action. In T. Karsenti et L. Savoie-Zajc (dir.), La recherche en éducation: étapes et approches (p. 181-208). Sherbrooke : Éditions du CRP.

Fortin, M.-F. (dir.) (2006). Fondements et étapes du processus de recherche. Montréal : Chenelière Éducation.

Fraenkel, J. et Wallen, N. (2003). How to Design and Evaluate Research in Education (5édition). New York, NY: McGraw-Hill. (1 ${ }^{\text {re }}$ éd. 1990)

Gagné, G., Lazure, R., Sprenger-Charolles, L. et Ropé, F. (1989). Recherches en didactique et acquisition du français langue matemelle - Tome 1. Bruxelles : De Boeck.

Gauthier, B. (2003). L'évaluation de la recherche par sondage. In B. Gauthier (dir), Recherche sociale: de la problématique à la collecte des données (4e édition) (p. 561-601). Québec: Presses de 1'Université du Québec. (1e éd. 1984)

Goigoux, R. (2002). Analyser l'activité d'enseignement de la lecture : une monographie. Revue française de pédagogie, $138,125-134$

Lebrun, J., Lenoir, Y., Oliveira, A. et Chalghoumi, H. (2005). La recherche sur les pratiques enseignantes effectives au préscolaire et au primaire. In $\mathrm{C}$. Gervais et $\mathrm{L}$. Portelance (dir), Des savoirs au cour de la profession enseignante (p. 265-285). Sherbrooke: Éditions du CRP.

Lefeuvre, G. (2005). L'accès aux pratiques d'enseignement à partir d'une double lecture de l'action. Journal International sur les Représentations Sociales, 2(1), 78-88.

Lenoir, Y. (2006). Du curriculum formel au curriculum enseigné. Conférence, FAPSE, Université de Genève.

Marcel, J.-F (2002). Le concept de contextualisation: un instrument pour l'étude des pratiques enseignantes. Revue française de pédagogie, 138, 103-113.

Marcel, J-F., Olry, P., Rothier-Bautzer, É. et Sonntag, M. (2002). Les pratiques comme objet d'analyse. Revue française de pédagogie, 138, 135-170.

Mialaret, G. (2004). Les méthodes de recherche en sciences de l'éducation. Paris : Presses universitaires de France.

Miles, M. et Huberman, A. (2003). Analyse des données qualitatives. Paris : De Boeck.

Ndouna Nsondé, Y. M. (2005). Les représentations de trois enseignantes de première année de Brazzaville par rapport à l'enseignement de la lecture. Thèse de doctorat, Université de Montréal, Québec.

Oliveira, A. (2005). Tendances actuelles de la recherche brésilienne portant sur les pratiques enseignantes: analyse critique de la production scientifique, 1985-2004. Mémoire de maîtrise, Université de Sherbrooke, Québec.

Roy, S. (2003). L'étude de cas. In B. Gauthier (dir.), Recherche sociale: de la problématique à la collecte des données (4édition) (p. 159-189). Québec: Presses de l'Université du Québec. (1e éd. 1984)

Saujat, F. (2002). Quand un professeur des écoles débutant instruit son «sosie» de son expérience... Les Dossiers des Sciences de l'Éducation, 7, 107-117.

Savoie-Zajc, L. (2001). La recherche-action en éducation. In M. Anadón (dir.), Nouvelles dynamiques de recherche en éducation (p. 15-49). Québec : Les Presses de l’Université Laval.

Savoie-Zajc, L. (2003). L'entrevue semi-dirigée. In B. Gauthier (dir.), Recherche sociale: de la problématique à la collecte des données (4édition) (p. 293-316). Québec: Presses de l'Université du Québec. (1 ${ }^{\text {re }}$ éd. 1984)

Savoie-Zajc, L. (2004). La recherche qualitative/interprétative en éducation. In T. Karsenti et L. Savoie-Zajc (dir), La recherche en éducation: étapes et approches (p. 123-150). Sherbrooke : Éditions du CRP.

Savoie-Zajc, L. et Karsenti, T. (2004). La méthodologie. In T. Karsenti et L. Savoie-Zajc (dir.), La recherche en éducation: étapes et approches (p. 109-121). Sherbrooke : Éditions du CRP.

Suchaut, B. (2003). De la nécessité d'évaluer les pratiques enseignantes: entre enjeux sociaux et obstacles méthodologiques. Les Dossiers des Sciences de l'Éducation, 10, 17-29. 
Tardif, M. et Lessard, C. (1999). Le travail enseignant au quotidien. Québec: Les Presses de l'Université Laval.

Terrisse, A. (2002). Entre unicité et pluralité: une synthèse impossible. In J.-F. Marcel (dir.), Les sciences de l'éducation: des recherches, une discipline (p. 233-250). Paris : L'Harmattan.

Tupin, F. (2003). Jalons pour une problématique générale. Les Dossiers des Sciences de l'Éducation, 10, 5-15.

Van der Maren, J.-M. (1996). Méthodes de recherche pour l'éducation (2 édition). Bruxelles: De Boeck. (1 ${ }^{\text {re }}$ éd. 1995)

Van der Maren, J.-M. (1999). La recherche appliquée en pédagogie: des modèles pour l'enseignement. Bruxelles: De Boeck. 
